\title{
Mini-Review: The Non-Immune Functions of Toll-Like Receptors
}

\author{
Ying Song, ${ }^{a},{ }^{*}$ Li Meng Shou, ${ }^{a, b}$ Li-Yao Ai, ${ }^{a}$ Yun Bei, ${ }^{a} \&$ Man-Ting Chena \\ aDepartment of Pharmacology, Zhejiang University of Technology, 18\# Chaowang Road, Hangzhou City, Zhejiang \\ Province 310014, P.R. China; 'The Second Affiliated Hospital, Zhejiang University School of Medicine (SAHZU) \\ SAHZU International Medical Center, No. 590, North Shixin Road, XiaoShao District, Hangzhou City, Zhejiang Province \\ 310009, P.R. China \\ *Address all correspondence to: Ying Song, Department of Pharmacology, Zhejiang University of Technology; Hangzhou Chaowang Road 18\#, \\ Zhejiang 310014, P.R. China; Tel./Fax: +0086-0571-88320776, E-mail: songying@zjut.edu.cn
}

\begin{abstract}
Toll-like receptors (TLRs) are a family of highly conserved pattern recognition receptors that can recognize both pathogen-associated molecular patterns and danger-associated molecular patterns. These receptors are important in the activation of the innate immune system and play a role in shaping the adaptive immune system. For years, the expression of TLRs in the brain has been proposed to contribute to the immunological protection of the central nervous system. However, emerging studies have provided increasing evidence of non-immune functions of TLRs and suggest that these receptors may participate in more complex processes that extend beyond the regulation of the innate immune response. In this review, we highlight the expression of TLRs in non-immune cells and epitomize TLR non-immune functions. We aim to reveal the novel roles of TLRs that are distinct from their traditional functions in immunity. Negative regulatory approaches used to study TLR signaling pathways are also discussed, providing potential directions for further studies.
\end{abstract}

KEY WORDS: Toll-like receptor, central nerve system, DNA repair, apoptosis, non-immune functions

\begin{abstract}
ABBREVIATIONS: AD, Alzheimer's disease; AP-1, activator protein-1; BrdU, bromodeoxyuridine; CNS, central nervous system; CpG, cytosine-phosphate-guanine; CREB, cAMP response element-binding; DAMP, danger-associated molecular pattern; ERK, extracellular signal-regulated kinase; IL, interleukin; LPS, lipopolysaccharide; MAL, MyD88 adaptor like; MyD88, myeloid differentiation 88; NER, nucleotide excision repair; NF- $\mathbf{B}$, nuclear factor-kappa beta; NPC, neural progenitor cell; PAMP, pathogen-associated molecular pattern; SARM, selective androgen receptor modulator; TRAM, TRIF-related adaptor molecule; TRIF, TIR-domain-containing adapter-inducing interferon- $\beta$
\end{abstract}

\section{INTRODUCTION}

Toll-like receptors (TLRs) are the best-studied pattern recognition receptors in mammals and play a role in sensing a wide array of pathogen-associated molecular patterns (PAMPs), including peptidoglycan (PGN), lipopolysaccharide (LPS), bacterial DNA, and double-stranded RNA. ${ }^{1}$ In addition, TLRs also respond to danger-associated molecular patterns (DAMPs) ${ }^{2}$ such as heat-shock proteins, chromatinprotein complexes, oxidized low-density lipoproteins, amyloid- $\beta$, and other factors (Table 1). ${ }^{3}$ Toll was first identified in Drosophila and was reported to be involved in the process of fungal infection, which established the immunological function of orthologous TLRs are widely expressed in immune cells. ${ }^{4}$
To date, the TLR family has been identified in most species, including vertebrates and invertebrates. For example, there are 10 TLRs (TLR1-TLR10) from humans and 12 TLRs (TLR1-TLR9 and TLR11TLR13) from mice. ${ }^{3}$

The location of TLRs is dependent on their PAMP recognition functions. Some TLRs (TLR1, TLR2, TLR4, TLR5, and TLR6) anchor onto the cell membrane to defend against extracellular pathogens, whereas other TLRs (TLR3, TLR7, TLR8, TLR9, TLR11, and TLR13) are expressed in the endosome and detect intracellular pathogens (Table 1). ${ }^{5}$ The structure of TLRs consists of three components: leucine-rich repeats (an endosomal pathogen-sensing domain), a transmembrane domain, and an intracellular Toll/interleukin-1 receptor (TIR) domain, 
TABLE 1: Comparisons of TLRs, including positions, PAMPs, DAMPs and signal adaptors

\begin{tabular}{|l|l|l|l|l|}
\hline Receptor & Position & PAMPs & DAMPs & Adaptor \\
\hline TLR1 & Cell surface & $\begin{array}{l}\text { Mycobacterium, } \\
\text { lipoprotein, triacyl lipid } \\
\text { peptide }\end{array}$ & Not determined & MAL/MyD88 \\
\hline TLR2 & Cell surface & $\begin{array}{l}\text { Lipopeptide, lipoprotein, } \\
\text { LTA, LAM }\end{array}$ & $\begin{array}{l}\text { HSP, mRNA, Hyaluronic } \\
\text { acid, Fibrinogen, HMGB-1 }\end{array}$ & MyD88 \\
\hline TLR3 & endosomal & dsRNA (polyI:C) & $\begin{array}{l}\text { HSP, mRNA, Hyaluronic } \\
\text { acid, Fibrinogen }\end{array}$ & TRIF \\
\hline TLR4 & Cell surface & LPS & $\begin{array}{l}\text { HSP, Hyaluronic acid, } \\
\text { HMGB-1, Heparan sulfate }\end{array}$ & MAL/MyD88 \\
& & & TRAM/TRIF \\
\hline TLR5 & Cell surface & Bacterial flagellin & Not determined & MyD88 \\
\hline TLR6 & Cell surface & Not determined & lipoprotein & MyD88 \\
\hline TLR7 & endosomal & ssRNA, dsRNA & ssRNA & MyD88 \\
\hline TLR8 & endosomal & ssRNA, dsRNA & ssRNA & MyD88 \\
\hline TLR9 & endosomal & dsRNA, CpG DNA & Chromatin IgG complex & MyD88 \\
\hline TLR10 & Cell surface & Profilin-like molecule & Not determined & MyD88 \\
\hline TLR11 & endosomal & Toxoplasma penicillin & Not determined & MyD88 \\
\hline TLR2/TLR1 & Cell surface & Lipopeptide & HSP, HMGB-1 & MAL/MyD88 \\
\hline TLR2/TLR6 & Cell surface & Lipopeptide & HSP, HMGB-1, ECM & MAL/My88 \\
\hline
\end{tabular}

The location of TLRs depends on their PAMP recognition functions. Some TLRs (TLR1, TLR2, TLR4, TLR5, and TLR6) anchor on the cell membrane and are in charge of defending against extracellular pathogens, whereas others (TLR3, TLR7, TLR8, TLR9, and TLR11) are expressed in the endosome to detect intracellular pathogens. TLRs sense a wide array of PAMPs, including LPS, bacterial DNA, and double-stranded RNA (dsRNA) (Anwar et al. ${ }^{3}$ ). In addition, TLRs also respond to DAMPs, such as heatshock proteins, chromatin-protein complexes, oxidized low-density lipoprotein, and amyloid- $\beta$. MyD88, MAL, TRIF, TRAM, and SARM are five adaptor proteins necessary for signaling transmission. MyD88 is the most popular adaptor required by all TLRs except TLR3 which uses TRIF as its sole adaptor molecule to trigger downstream signaling.

HSP, heat shock protein; LAM, lipoarabinomannan; LTA, lipoteichoic acid; HMGB-1, high mobility group box-1 protein; ssRNA, single-stranded RNA; ECM, extracellular matrix; IgG, immunoglobin G.

which distinguishes between various TLR family members and interacts with signaling or adaptor molecules to relay information about the detected pathogen. ${ }^{6}$ Myeloid differentiation 88 (MyD88), MyD88 adaptor like (MAL), TIR-domain-containing adapter-inducing interferon- $\beta$ (TRIF), TRIF-related adaptor molecule (TRAM), and selective androgen receptor modulator (SARM) are five adaptor pro- teins necessary for signal transmission. MyD88 is the best known adaptor and is required by all TLRs except TLR3, which uses TRIF as its sole adaptor molecule to trigger downstream signaling. All TIR adaptors have important positive roles in mediating intracellular signaling following TLR stimulation except SARM, which has been reported to inhibit TRIF-dependent TLR signaling (Table 1). ${ }^{7}$ 


\section{NEGATIVE REGULATORY APPROACHES TO TLR SIGNALING PATHWAYS}

Increasing evidence shows new characteristics of TLRs. For instance, the ability of TLRs to sense DAMPs make them ideal candidates for the overarching regulation of DNA repair processes. However, how do TLRs differentiate signals upregulating DNA repair from signals that induce a tissue-destructive inflammatory response? Moreover, how does one find a balance between these two processes? Identifying the molecules involved in the repair process induced by TLRs is crucial for better understanding TLR functioning. Even if TLRs are indispensable in the induction of the innate immune response and the enhancement of adaptive immunity against pathogens, sustained inflammatory signaling can be toxic and activation of TLRs may result in excessive inflammation and autoimmune diseases. ${ }^{8}$ The TLR signaling pathway must therefore be tightly regulated.

Subsequent to stimulation with their cognate ligands, TLRs induce several mediators converging

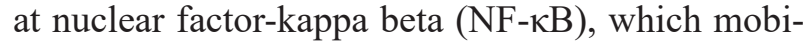
lizes the transcription of inflammatory genes. This process helps the host defend against the microbial threat. After eliminating the threat, the cell must turn off the expression of inflammatory mediators. Sustained activation and overactivation of TLRs are usually accompanied by deleterious consequences to the host. To prevent these consequences, the cell employs a variety of mechanisms to regulate TLR signaling, including decoy factors, adaptor modification, ubiquitin-mediated degradation, promoter state alteration, and translation disruption. These mechanisms, individually or in combination, control inflammation and allow the system to return to its normal state. ${ }^{3}$

\section{TLR SIGNALING INDUCES DNA REPAIR}

Klaschi ${ }^{9}$ performed global gene expression analysis on mice treated with TLR9 ligand: cytosine-phosphate-guanine $(\mathrm{CpG})$ DNA to investigate changes (upregulated or downregulated expression) on various gene families in spleen cells. ${ }^{10}$ They found a signifi- cant increase in mouse splenic mRNAs that code for proteins involved in the regulation of cell cycle and DNA repair 5 days after intraperitoneal injection of CpG DNA. Similar results were also found in an in silico gene expression study using datasets obtained after intraperitoneal injection of CpG DNA as well as intranasal inoculation. They found that genes encoding for DNA repair molecules were upregulated in immune cells. ${ }^{11}$ Moreover, imiquimod, an agonist of TLR7 and TLR8, was found by Fishelevich to enhance DNA repair gene expression as well as functional repair of DNA damage mechanisms in UV-irradiated mice. ${ }^{12,13}$ Nucleotide excision repair (NER) gene expression is upregulated in addition to the nuclear localization of the repair molecule, xeroderma pigmentosum complementation group $\mathrm{A}$ in a bone-marrow-derived cell line treated with imiquimod. In vivo studies have demonstrated enhanced resolution of cyclobutane pyrimidine dimmers in UV-irradiated and Imiquimod-treated mice compared with control-irradiated mice. Taken together, these results indicate that TLR signaling may be related to DNA repair and contribute to growing evidence for non-immune functions of TLRs. Furthermore, it has been found that peripheral blood mononuclear cells from xeroderma pigmentosum patients have reduced interferon-gamma production following stimulation with polyinosinic:polycytidylic acid (polyI:C), a TLR3 agonist, ${ }^{14}$ which suggest impaired TLR3 signaling. TLR3 uses the TRIF and MyD88 pathways to upregulate DNA repair. In contrast to healthy lymphoblasts, lymphoblasts deficient in DNA repair failed to augment DNA repair after MyD88 knockdown following UV radiation, which may suggest that the TLR4/MyD88 pathway is a useful tool in promoting DNA repair and maintaining immune responses following UV radiation-induced damage. ${ }^{15}$

The role of TLRs in DNA repair remains elusive. As mentioned in the introduction section, TLRs respond to DAMPs, which are produced under conditions of oxidative stress and DNA damage. Many DAMPs that could be recognized by TLRs, such as self-DNA binding with proteins, self-noncoding RNAs, and some histone proteins, may serve as a DNA damage signal to improve cellular DNA repair machine. But how do TLRs induce DNA repair? The 
possible mechanism by which TLRs induce DNA repair may function in two ways. ${ }^{16}$ One mechanism may involve the presence of the activator protein-1 (AP-1)-binding site on promoter regions of genes. AP-1, a heterodimeric protein composed of c-Fos and c-Jun, is a transcription factor. Based on computer analysis, the promoter regions of NER genes contain at least one AP-1-binding site. ${ }^{17}$ The production of AP-1 induced by TLRs and the presence of AP-1-binding sites on DNA repair genes create a bridge between TLR signaling and DNA repair. Another potential mechanism involves autocrine or paracrine of cytokines induced by TLRs. Many cytokines, including interleukin-12 (IL-12), IL-18, and IL-23, stimulate DNA repair mechanisms, which results in increased functional repair. ${ }^{18}$ These possible mechanisms need to be further supported by direct experimental evidence (Fig. 1). ${ }^{19}$

\section{TLRS REGULATE CELL FATE (PROLIFERATION AND DIFFERENTIATION)}

TLRs are not only expressed on antigen-presenting cells; many are also expressed by neurons, microglial cells, and astrocytes. ${ }^{20}$ Importantly, the expression level of TLRs is not immutable in development. In early embryos, TLR8 is highly expressed in peripheral sensory and sympathetic ganglia and in postmitotic migrating central nervous system (CNS) cells, but not in the periventricular cell proliferation zone. Furthermore, in late embryonic brains, TLR8 expression is restricted to axonal tracts, including the olfactory nerve fiber layer, cortical intermediate zone, internal capsule, anterior commissure, fimbria of the hippocampus, optic chiasm, and other major fiber systems. Postnatally, TLR8 is diffusely expressed in most brain regions and localizes mainly to neuronal somata. ${ }^{21}$ This dynamically changing spatiotemporal expression pattern indicates that TLR8 may participate in brain development. In addition, agonist (R-848) stimulation of TLR8 in cultured cortical neurons inhibits neurite outgrowth and induces apoptosis in a dissociable manner. There is evidence that suggests R-848 stimulation of TLR8 does not induce canonical NF- $\mathrm{kB}$ transactivation in cultured neurons.
I $\mathrm{B} \alpha(\mathrm{Ser} 32)$ phosphorylation and other characteristic hallmarks of TLR signaling, including phosphorylation of extracellular signal-regulated kinases 1 and 2 (ERK1/2), SAPK/JNK, Akt, and GSK3 $\beta$, are not detected in neurons. ${ }^{21}$ Taken together, these findings support a new function of TLR8 in neurons that is not dependent on its conventional immune pathway, the NF- $\kappa \mathrm{B}$ cascade.

In addition, TLR4 increases the number of neocortical neurons after stroke and plays an important role in stroke-induced neurogenesis. ${ }^{22}$ Absence of TLR4 leads to enhanced neuronal differentiation and TLR4 contributes to the growth of axon. Experiments have shown that the lack of TLR4 leads to impaired neo-axonal growth by the TLR4 pathway that plays an important role in axon debris clearance, which provides a more relaxed environment for axonal growth. Therefore, TLR4-dependent clearance of unmyelinated axon fragments promotes axonal growth. ${ }^{23}$

Similar findings have been observed in TLR3. Neurons in the mature CNS are able to grow new axons in permissive environments. However, axons have a limited ability to regenerate during CNS injury. Activation of TLR 3 by polyI:C or by mRNA rapidly causes growth cones to collapse and irreversibly inhibits neurite extension independent of NF- $\mathrm{kB} .{ }^{24}$ These results demonstrate that TLR3 also functions as a negative regulator of axonal growth in mammals despite being an immune pattern recognition receptor.

Many other studies have also investigated the non-immune characteristics of TLRs. TLR2 is expressed in the developing mouse brain and in cultured embryonic neural progenitor cells (NPCs). Although its expression does not affect NPC proliferation in vitro or in vivo, its activation inhibits NPC proliferation in vitro and decreases cell proliferation in vivo. Activation of TLR2 with bacterial ligands in utero results in ventricular dysplasia in embryonic mice. ${ }^{25}$ It has been reported that TLR3 also negatively regulates embryonic NPC proliferation during development and therefore controls the growth of the developing cerebral cortex. Moreover, TLR3 protein is present in brain cells in early embryonic stages of development and in cultured NPCs. NPCs from TLR3-deficient embryos formed a greater number of 


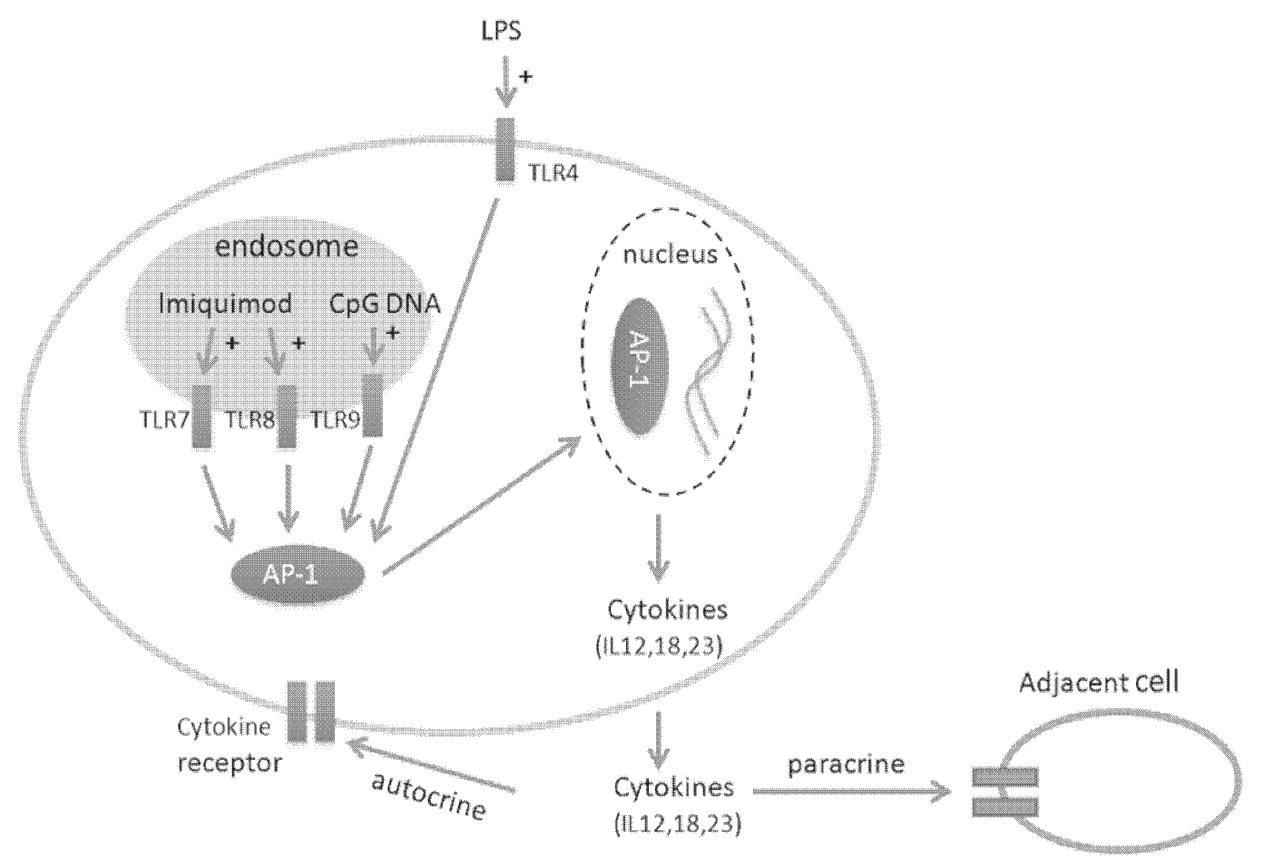

FIG. 1: Mechanisms of DNA repair by TLR signaling. TLR signaling induces AP-1 by DAMP stimulation of TLR generated under oxidative stress and DNA damage. This TLR-induced production of AP-1 and the presence of the AP1-binding site on the DNA repair gene achieve DNA repair. The autocrine or paracrine secretion of cytokines induced by TLR signaling stimulate DNA repair mechanisms and lead to increased functional DNA repair.

neurospheres compared with neurospheres derived from wild-type embryos. The number of proliferating cells, as assessed by phosphor-histone $\mathrm{H} 3$ and proliferating cell nuclear antigen labeling, was also increased in the developing cortex of TLR3-deficient mice compared with wild-type mice in vivo. Treatment of cultured embryonic cortical neurospheres with a TLR3 ligand (polyI:C) significantly reduced proliferating (bromodeoxyuridine [BrdU]-labeled) cells and neurosphere formation in wild-type but not in TLR3 ${ }^{-1-}$-derived NPCs. ${ }^{26}$ In addition, the role of TLRs in cell proliferation is not limited to NPCs. The activation of TLR4 by ultrapurified LPS has been suggested to be an intrinsic regulator, restricting both the proliferation and neural differentiation of retinal progenitor cells. ${ }^{27}$ That study found that downregulation of TLR4 can inhibit the excessive proliferation of mesangial cells by PI3K/AKT pathway. ${ }^{28}$ These findings demonstrate that TLRs play an important role in cell growth in addition to regulation of the immune response.

These studies have begun to unravel a previously unknown role of TLRs. However, the mechanisms underlying the regulation of proliferation, differentiation, and growth by TLRs are still unclear. The AP-1 theory may be a clue. The regulation of genes encoding or cytokines and growth factors can be triggered when AP-1 responds to stress, viral infection, and bacterial invasion. ${ }^{29}$ Numerous processes are regulated by AP-1, including differentiation, proliferation, and apoptosis. Does the production of AP-1 induced by TLRs serve as a link between TLRs and cell growth? This hypothesis requires further validation. It is difficult to identify TLRs' endogenous agonists that trigger the cascade of TLR signaling and finally decide the cell fate. Injury- or stress-induced DAMPs may be responsible for this process (Fig. 2). ${ }^{29}$ 


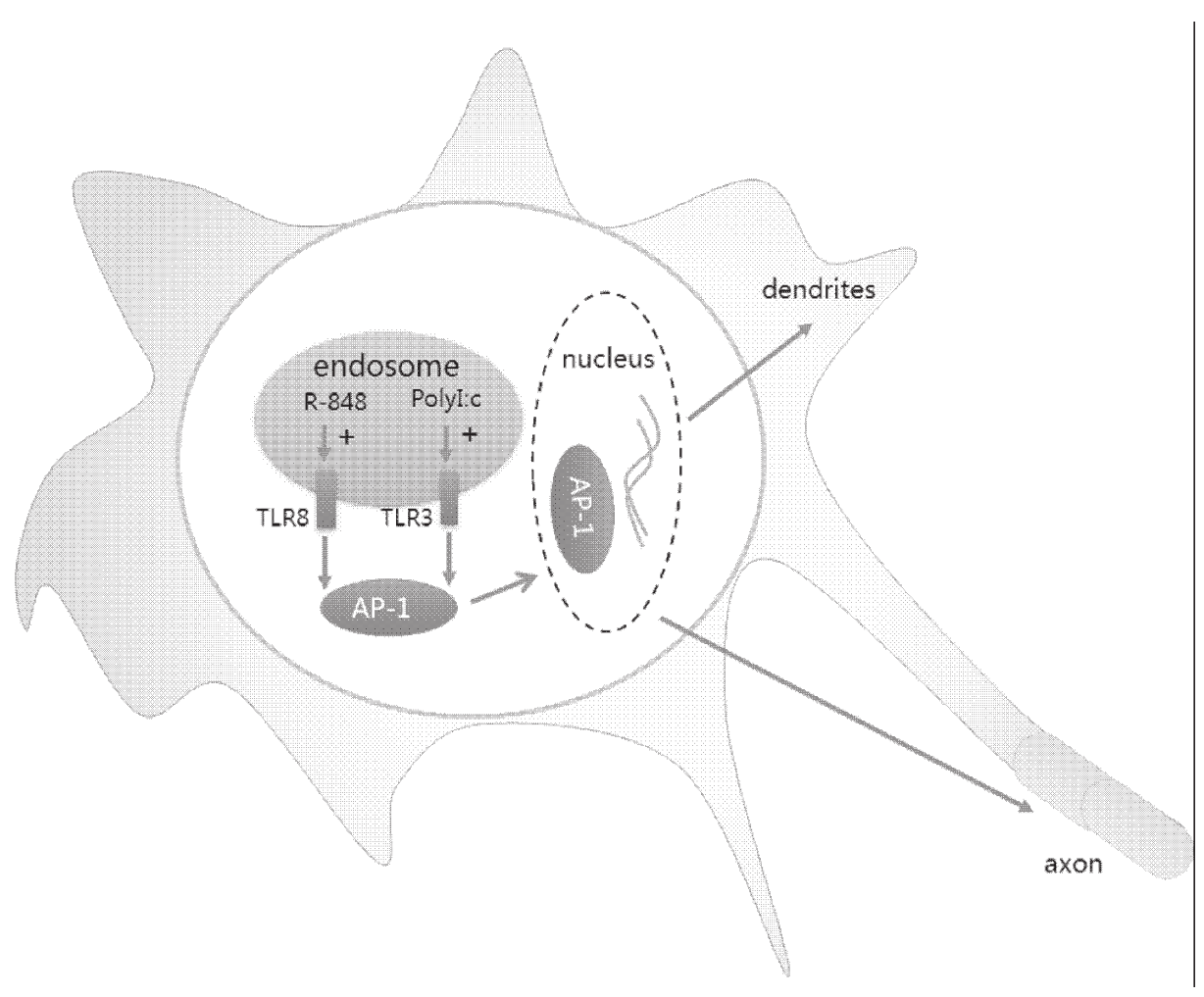

FIG. 2: Mechanism of cell fate regulated by TLRs in neurons. TLRs are expressed in neurons and induced TLR signaling through DAMPs stimulation to induce AP-1 production. Subsequently, AP-1 regulates the growth of neurites and axons.

\section{TLRS AND NON-INFECTIOUS DISEASES IN THE CNS}

Because TLRs are expressed on antigen-presenting cells such as B cells, monocytes, and macrophages, we take it for granted that TLRs serve as immune guardians in response to pathogens. However, several studies have reported that TLRs (on the mRNA or protein level) are expressed in nearly all cell types, even neurons in the CNS (Table 2). ${ }^{6}$ In addition, the dynamic expression patterns of TLRs that occur during brain development and during signaling independent of the NF- $\kappa \mathrm{B}$ pathway both indicate that there is a wealth of information to be revealed with regard to TLRs in brain development and homeostasis. Carty and Bowie ${ }^{6}$ examined the recent literature on TLRs in the CNS and demonstrated their importance in a range of brain diseases. Here, we performed a further summary of TLRs involved in non-infectious diseases in the CNS (Table 3). ${ }^{6}$
However, the corresponding mechanisms and the pathogenesis of some diseases are unknown. For example, Alzheimer's disease (AD) is a common neurodegenerative disease with an unknown pathogenesis. The accumulation of $A \beta$, which results in the development of extracellular senile plaques and intracellular neurofibrillary tangles, has been related to AD progression. Furthermore, TLR 4 has been shown to mediate neuronal apoptosis in response to $\mathrm{A} \beta,{ }^{30}$ suggesting a role of TLR4 in AD.

TLR3 was found to inhibit memory retention and to constrain adult hippocampal neurogenesis. A comparison between wild-type and TLR3-deficient mice ${ }^{31}$ revealed that TLR3 deficiency enhances hippocampus-dependent working memory but impairs amygdala-related behavior, indicating that TLR3 is a negative regulator of hippocampus-dependent learning and memory. Moreover, the hippocampus of TLR3-deficient mice shows a significant increase in BrdU-labeled cells co-expressing the mature neuronal 
TABLE 2: Expression of TLR in immune cells and CNS

\begin{tabular}{|l|l|l|l|}
\hline Molecule detected & \multicolumn{1}{|c|}{ CNS } & Immune cells & $\begin{array}{l}\text { Epithelial or } \\
\text { endothelial cells }\end{array}$ \\
\hline TLR1 & Microglia, astrocytes & $\begin{array}{l}\text { Mononuclear cells, TCs, BCs, NK cells, } \\
\text { DCs }\end{array}$ & Epithelial \\
\hline TLR2 & Microglia, astrocytes, neurons & Mononuclear cells, DCs, macrophages & Epithelial \\
\hline TLR3 & Microglia, astrocytes, neurons & Mononuclear cells, DCs & Both \\
\hline TLR4 & Microglia, astrocytes, neurons & Mononuclear cells, DCs, TCs, BCs & Both \\
\hline TLR5 & Microglia, astrocytes & Mononuclear cells, DCs & Epithelial \\
\hline TLR6 & Microglia, astrocytes & Mononuclear cells & Epithelial \\
\hline TLR7 & Microglia, astrocytes & $\begin{array}{l}\text { Mononuclear cells, DCs, TCs, BCs, NK } \\
\text { cells }\end{array}$ & Both \\
\hline TLR8 & Microglia, astrocytes & Mononuclear cells, DCs & Endothelial \\
\hline TLR9 & Microglia, astrocytes & $\begin{array}{l}\text { Mononuclear cells, DCs, TCs, BCs, } \\
\text { macrophages, NK cells }\end{array}$ & Epithelial \\
\hline
\end{tabular}

TLR is expressed in most cells (Anwar et al. ${ }^{3}$ ). TLRs are expressed not only in the immune system, including mononuclear cells, thymus-dependent lymphocytes (TCs), B lymphocytes (BCs), and natural killer (NK) cells, but also in the CNS, including microglia, astrocytes, and neurons. Different TLRs are expressed in different cells.

TABLE 3: TLRs in non-infectious diseases

\begin{tabular}{|l|l|l|}
\hline Non-infectious diseases & Mouse TLRs implicated & Human TLRs implicated \\
\hline AD & TLR2, TLR4, CD14 & $\begin{array}{l}\text { TLR4 polymorphism Asp299Gly, CD14 } \\
\text { increased in senile plaques }\end{array}$ \\
\hline Experimental autoimmune encephalitis & MyD88, TLR4, TLR9 & \\
\hline Multiple sclerosis & TLR2, TLR4 & Increased TLR3 and TLR4 \\
\hline Stroke & TLR2, TLR4 & TLR4 polymorphism, C119A \\
\hline Glioma & TLR9 & $\begin{array}{l}\text { TLR9 expression evaluated in Phase 1 and } \\
\text { Phase 2 clinical trials }\end{array}$ \\
\hline
\end{tabular}

TLRs are involved in many non-infectious diseases in the CNS, such as $\mathrm{AD}$, multiple sclerosis, and glioma (Carty and Bowie $\left.{ }^{6}\right)$. TLRs are important in AD, in particular those TLRs that are expressed in microglia. For example, animal models of AD and patients with AD exhibit increased expression of CD14 (a co-receptor for TLR4), TLR4, and TLR2.

maker NeuN, corresponding to increased neuronal differentiation and/or cell survival. These results also show that the canonical TLR is not altered in TLR3 ${ }^{-/}$ mice. However, the levels of phosphorylated ERK and phosphorylated cAMP response element-binding (CREB) are elevated in the hippocampus of TLR3 $3^{-/-}$ mice compared with $\mathrm{TLR}^{+/+}$mice, demonstrating that the presence of TLR3 suppresses the ERK and CREB signaling pathway. The new function of TLR3 in adult CNS plasticity and behavior implicates its involvement in neurophysiology and neuropathology, which may be similar to the role of TLR4 in

\section{AD progression. ${ }^{30}$}

\section{FUTURE PROSPECTS}

Thus far, TLRs have been shown to function not only the immune system, but also many more complex cell processes. Its non-immune functions have also been demonstrated in numerous studies. However, the precise mechanism of such functions has not yet been elucidated. Therefore, additional studies are required to elucidate the molecules that contribute 
to this specific signaling pathway. Do different subtypes of TLRs share the same expression pattern and function in non-immune system? Do they regulate in the same manner? These questions remain to be solved. New findings in further studies on the precise mechanism of TLRs' non-immune functions will provide a better understanding of TLRs as well as unique opportunities for the application of TLR signaling in relevant biological areas.

\section{ACKNOWLEDGMENTS}

This work was supported by the Natural Science Foundation of Zhejiang Province (Grant LY17H090018) and the National Special Foundation for Clinical Medicine Research (Grant L2011079).

\section{REFERENCES}

1. Kanwal Z, Wiegertjes GF, Veneman WJ, Meijer AH, Spaink HP. Comparative studies of Toll-like receptor signalling using zebrafish. Develop Comp Immunol. 2014;46(1):35-52.

2. Prince LR, Whyte MK, Sabroe I, Parker LC. The role of TLRs in neutrophil activation. Curr Opin Pharmacol. 2011;11(4):397-403.

3. Anwar MA, Basith S, Choi S. Negative regulatory approaches to the attenuation of Toll-like receptor signaling. Exp Mol Med. 2013;45:e11.

4. Lemaitre B, Nicolas E, Michaut L, Reichhart JM, Hoffmann JA. The dorsoventral regulatory gene cassette spätzle/Toll/cactus controls the potent antifungal response in Drosophila adults. Cell. 1996;86(6):973-83.

5. Chen C, Zibiao H, Ming Z, Shiyi C, Ruixia L, Jie W, SongJia L. Expression pattern of Toll-like receptors (TLRs) in different organs and effects of lipopolysaccharide on the expression of TLR 2 and 4 in reproductive organs of female rabbit. Dev Comp Immunol. 2014;46(2):341-8.

6. Carty M, Bowie AG. Evaluating the role of Toll-like receptors in diseases of the central nervous system. Biochem Pharmacol. 2011;81(7):825-37.

7. Carty M, Goodbody R, Schroder M, Stack J, Moynagh PN, Bowie AG. The human adaptor SARM negatively regulates adaptor protein TRIF-dependent Toll-like receptor signaling. Nat Immunol. 2006;7(10):1074-81.

8. Panter G, Jerala R. The ectodomain of the Toll-like receptor 4 prevents constitutive receptor activation. J Biol Chem. 2011;286(26):23334-44.

9. Klaschik S, Tross D, Shirota H, Klinman DM. Short- and long-term changes in gene expression mediated by the activation of TLR9. Mol Immunol. 2010;47(6):1317-24.

10. Liqin Z, Nicole A, Angela HK, Paulo R, Kevin DB, Eduardo D. TLR9 engagement on CD4 T lymphocytes represses $\gamma$-radiation-induced apoptosis through activation of checkpoint kinase response elements. Blood. 2008;111:2704-13.

11. Sommariva M, De Cecco L, De Cesare M, Sfondrini L, Menard S, Melani C, Delia D, Zaffaroni N, Pratesi G, Uva V, Tagliabue E, Balsari A. TLR9 agonists oppositely modulate DNA repair genes in tumor versus immune cells and enhance chemotherapy effects. Cancer Res. 2011;71(20):6382-90.

12. Fishelevich R, Zhao Y, Tuchinda P, Liu H, Nakazono A, Tammaro A, Meng TC, Lee J, Gaspari AA. Imiquimodinduced TLR7 signaling enhances repair of DNA damage induced by ultraviolet light in bone marrow-derived cells. J Immunol. 2011;187(4):1664-73.

13. Larange A, Antonios D, Pallardy M, Kerdine-Romer S. TLR7 and TLR8 agonists trigger different signaling pathways for human dendritic cell maturation. J Leukoc Biol. 2009;85(4):673-83.

14. Gaspari AA, Fleisher TA, Kraemer KH. Impaired interferon production and natural killer cell activation in patients with the skin cancer-prone disorder, xeroderma pigmentosum. J Clin Invest. 1993;92(3):1135-42.

15. Erin H, Hua Z, Rita F, Juan Liu, Gaspari. Ultraviolet radiation signaling through TLR4/MyD88 constrains DNA repair and plays a role in cutaneous immunosuppression. J Immunol. 2015;197(7):3127-35.

16. Harberts E, Gaspari AA. TLR signaling and DNA repair: are they associated? J Invest Dermatol. 2013;133(2):296302.

17. Zhong X, Thornton K, Reed E. Computer based analyses of the 5'-flanking regions of selected genes involved in the nucleotide excision repair complex. Int J Oncol. 2000;17(2):375-80.

18. Majewski S, Jantschitsch C, Maeda A, Schwarz T, Schwarz A. IL-23 antagonizes UVR-induced immunosuppression through two mechanisms: reduction of UVR-induced DNA damage and inhibition of UVR-induced regulatory T cells. J Invest Dermatol. 2010;130(2):554-62.

19. Harberts E, Gaspari AA. TLR signaling and DNA repair: are they associated? J Invest Dermatol. 2013;133(2):296302.

20. Larsen PH, Holm TH, Owens T. Toll-like receptors in brain development and homeostasis. Sci STKE. 2007;2007(402):pe47.

21. Ma Y, Li J, Chiu I, Wang Y, Sloane JA, Lu J, Kosaras B, Sidman R, Volpe J, Vartanian T. Toll-like receptor 8 functions as a negative regulator of neurite outgrowth and inducer of neuronal apoptosis. J Cell Biol. 2006;175(2):209-15.

22. Ana M, Jesús MP, María IC, Macarena HJ, Marta O, María AM, Ignacio L. Toll-like receptor 4 modulates cell 
migration and cortical neurogenesis after focal cerebral ischemia. FASEB J. 2014;28(11):4710-8.

23. Labchan R, Million AT, Shiva S, Nishant GK, Adeel M, Aditya M, Suneil H, Arun V. Toll-like receptor 4 deficiency impairs microglial phagocytosis of degenerating axons. Res Article. 2014;62(12):1982-91.

24. Cameron JS, Alexopoulou L, Sloane JA, DiBernardo AB, Ma Y, Kosaras B, Flavell R, Strittmatter SM, Volpe J, Sidman R, Vartanian T. Toll-like receptor 3 is a potent negative regulator of axonal growth in mammals. $\mathrm{J}$ Neurosci. 2007;27(47):13033-41.

25. Eitan O, Kathleen JG, Mark PM. Toll-like receptor signaling in neural plasticity and disease. Trends Neurosci. 2011;34(5):269-81.

26. Lathia JD, Okun E, Tang SC, Griffioen K, Cheng A, Mughal MR, Laryea G, Selvaraj PK, Ffrench-Constant C, Magnus T, Arumugam T, Mattson MP. Toll-like receptor 3 is a negative regulator of embryonic neural progenitor cell proliferation. J Neurosci. 2008;28(51):13978-84.

27. Okun E, Griffioen K, Barak B, Roberts NJ, Castro K, Pita MA, Cheng AW, Mughal MR, Wan RQ, Ashery U, Mattson MP. Toll-like receptor 3 inhibits memory retention and constrains adult hippocampal neurogenesis. Proc Natl Acad Sci U S A. 2010;107(35):15625-30.

28. Tao D, Wei C, Juan L, Jiarong D, Haiyan H, Xiaobin M. High glucose induces mouse mesangial cell overproliferation via inhibition of hydrogen sulfde synthesis in a TLR-4-dependent manner. Cell Physiol Biochem. 2017;41(3):1035-43.

29. Kutuzov MM, Khodyreva SN, Ilina ES, Sukhanova MV, Ame JC, Lavrik OI. Interaction of PARP-2 with AP site containing DNA. Biochimie. 2015;112:10-9.

30. Tang SC, Lathia JD, Selvaraj PK, Jo DG, Mughal MR, Cheng A, Siler DA, Markesbery WR, Arumugam TV, Mattson MP. Toll-like receptor-4 mediates neuronal apoptosis induced by amyloid beta-peptide and the membrane lipid peroxidation product 4-hydroxynonenal. Exp Neurol. 2008;213(1):114-21.

31. Okun E, Griffioen KJ, Son TG, Lee JH, Roberts NJ, Mughal MR, Hutchison E, Cheng AW, Arumugam TV, Lathia JD, van Praag H, Mattson MP. TLR2 activation inhibits embryonic neural progenitor cell proliferation. J Neurochem. 2010;114(2):462-74.

32. Al-Sadi R, Nighot P, Guo S, Al-Omari D, Ma TY. Tu2019 Lactobacillus acidophilus enhancement of intestinal epithelial tight junction barrier is mediated by $\mathrm{p} 38$ kinase and Toll-like receptor-2 (TLR-2). Gastroenterology.
2016;150(4):S1007.

33. Chassaing B, Gewirtz AT. Sa1723 role of intestinal epithelial cell, dendritic cell, and hepatocyte TLR5 in keeping the microbiota in-check and protecting the gut. Gastroenterology. 2014;146(5):S-281-2.

34. Dhamanage A, Thakar M, Paranjape R. Human immunodeficiency virus-1 impairs IFN-alpha production induced by TLR-7 agonist in plasmacytoid dendritic cells. Viral Immunol. 2017;30(1):28-34.

35. Holfeld J, Tepekoylu C, Mathes W, Lobenwein D, Kozaryn R, Krapf C, Urbschat A ,Kirchmair R, Grimm $\mathrm{M}$, Zacharowski K. Loss of regenerative response upon low energy shock waves in toll-like receptor 3 silenced endothelial cells and TLR-3 knock out mice in a hind limb ischemia model. Wiener Klinische Wochenschrift. 2013;125(1):S18.

36. Jing C, Yuefeng X, Karla W, Meihong D, Kory JB, David AV, Heth RT, Timothy RB, Bruce AP, Ma-Zhong Z, LiMing Z. Cyclic stretch induced IL-33 production through HMGB1/TLR-4 signaling pathway in murine respiratory epithelial cells. PLoS One. 2017;12(9):e0184770.

37. Koopman G, Beenhakker N, Burm S, Bouwhuis O, Bajramovic J, Sommandas V, Mudde G, Mooij P, 't Hart BA, Bogers WMJM. Whole blood stimulation with Toll-like receptor (TLR)-7/8 and TLR-9 agonists induces interleukin-12p40 expression in plasmacytoid dendritic cells in rhesus macaques but not in humans. Clin Exp Immunol. 2013;174(1):161-71.

38. Lang T, Mansell A. The negative regulation of Toll-like receptor and associated pathways. Immunol Cell Biol. 2007;85(6):425-34.

39. Moraga A, Pradillo JM, Cuartero MI, Hernandez-Jimenez M, Oses M, Moro MA, Lizasoain I. Toll-like receptor 4 modulates cell migration and cortical neurogenesis after focal cerebral ischemia. FASEB J. 2014;28(11):4710-8.

40. Wang S, Villablanca EJ, De Calisto J, Gomes DC, Nguyen DD, Mizoguchi E, Kagan JC, Reinecker HC, Hacohen N, Nagler C, Xavier RJ, Rossi-Bergmann B, Chen YB, Blomhoff R, Snapper SB, Mora JR. MyD88-dependent TLR1/2 signals educate dendritic cells with gut-specific imprinting properties. J Immunol. 2011;187(1):141-50.

41. Zheng L, Asprodites N, Keene AH, Rodriguez P, Brown KD, Davila E. TLR9 engagement on CD4 T lymphocytes represses gamma-radiation-induced apoptosis through activation of checkpoint kinase response elements. Blood. 2008;111(5):2704-13. 